\title{
Nonlinear Model Reduction and Decentralized Control of Tethered Formation Flight by Oscillation Synchronization
}

\author{
Soon-Jo Chung \\ *Massachusetts Institute of Technology, MA 02139, USA
}

\begin{abstract}
This paper describes a fully decentralized nonlinear control law for spinning tethered formation flight, based on exploiting geometric symmetries to reduce the original nonlinear dynamics into simpler stable dynamics. Motivated by oscillation synchronization in biological systems, we use contraction theory to prove that a control law stabilizing a single-tethered spacecraft can also stabilize arbitrary large circular arrays of spacecraft, as well as the three inline configuration. The convergence result is global and exponential. Numerical simulations and experimental results using the SPHERES testbed validate the exponential stability of the tethered formation arrays by implementing a tracking control law derived from the reduced dynamics.
\end{abstract}

\section{Introduction}

Spacecraft formation flight is becoming a key research area, where distributed computation and decentralized control schemes, as well as information flows between elements, are explored. One such example includes stellar interferometers in which multiple apertures in controlled formation collect light for coherent interferometric beam combination, thereby achieving a fine angular resolution comparable to a large monolithic aperture telescope. ${ }^{1}$ The possible architectures of spaceborne interferometers include a Structurally Connected Interferometer (SCI), which allows for very limited baseline changes, and a Separated Spacecraft Interferometer (SSI) ${ }^{2}$ where the usage of propellant can be prohibitively expensive. A tethered formation flight interferometer represents a balance between SCI and SSI. Such a system is currently being considered for NASA's Submillimeter Probe of the Evolution of Cosmic Structure (SPECS) mission. ${ }^{3,4}$ The dynamics of SSI are coupled by the definition of relative attitude whereas tethered formation spacecraft exhibit inherently coupled nonlinear dynamics. In spite of the popularity of these modular architectures, it is rare to find work on modular stability analysis for such coupled dynamics systems.

This paper describes a decentralized nonlinear control law for spinning tethered formation flight, based on exploiting geometric symmetries to reduce the original nonlinear dynamics into simpler stable dynamics. In dealing with such coupled dynamics, an attempt is made to learn from distributed computation in biological systems. Figure 1 shows a picture of a human running by synchronizing oscillations of his leg joints; a three-spacecraft tethered formation can also be viewed as a multiple-joint system. Networked arrays of coupled dynamics abound in biological systems. For example, motion control architecture in vertebrates involves combinations of simple motor primitives, ${ }^{6-8}$ and synchronous fireflies ${ }^{5}$ and animal gaits ${ }^{6,9}$ might shed some light on the stability of coupled arrays. Some recent works on multi-agent robots, ${ }^{10}$ aircraft formation, ${ }^{11,12}$ and robotics, ${ }^{13}$ also deal with coupled oscillations. In control theory, Popov's passivity ${ }^{16}$ was originally motivated by similar concerns. However, combinations and accumulations of stable elements do not necessarily result in stable dynamics. ${ }^{18}$ This paper uses contraction theory ${ }^{17-19}$ to analyze the nonlinear stability of a decentralized control of tethered formation flight. Contraction theory provides a systematic method to build arbitrarily complex systems (a tethered formation array) out of simpler elements (a singletethered spacecraft). Combinations validated by contraction theory, such as hierarchical combinations and

\footnotetext{
*Doctoral Student, Department of Aeronautics and Astronautics, and AIAA Student Member, sjchung@mit.edu. This work is done solely by the author under the supervision of Prof. Jean-Jacques Slotine and Prof. David W. Miller.
} 
synchronized coupled oscillations, are explored to simplify the nonlinear dynamics of multiple tethered spacecraft.

One benefit of a control law based upon such reduced dynamics is that we can implement simple decentralized $^{12,14}$ control and estimation algorithms for tethered formation flight systems. The decentralized controller will enable a simple independent control of each satellite without the need for exchanging information about individual states. This will significantly simplify both the control algorithm and hardware implementation as well as eliminating communication delays from such a large separation (e.g. $1 \mathrm{~km}$ for SPECS). Dynamics modeling in this paper facilitates a relative and decentralized sensing mechanism for
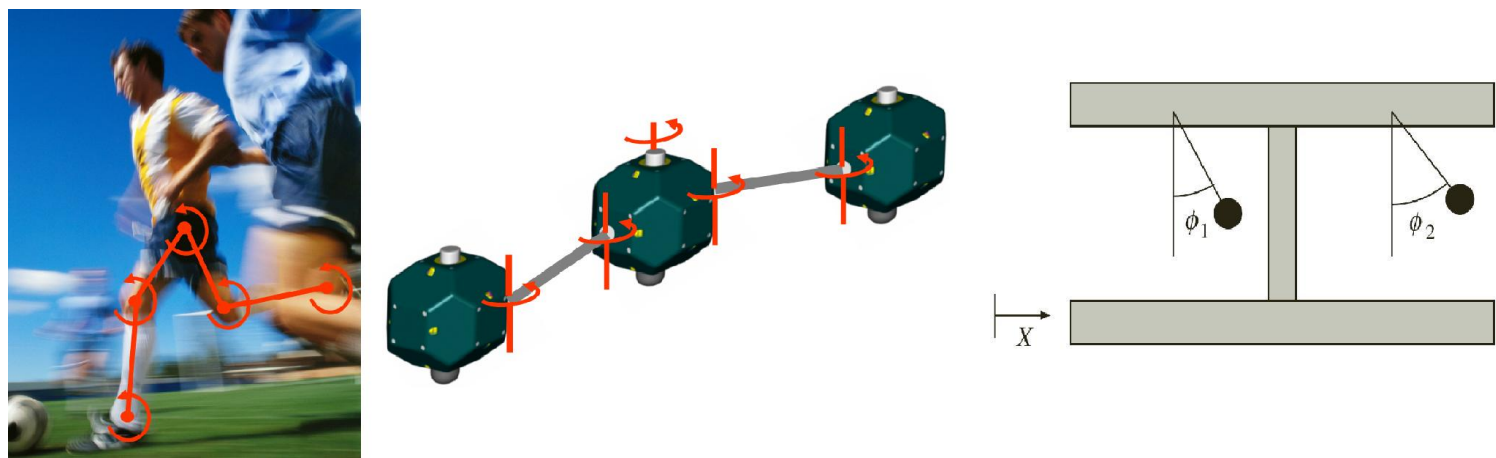

Figure 1. synchronization of motions: human locomotion (left), three tethered spacecraft (middle), and Huygens's pendulum clock $^{36}$ (right)

deep space formation flight. In deep space, absolute attitude might be available via star-trackers but the availability of absolute positions like those provided by Global Positioning System (GPS) is very limited. ${ }^{15}$

There are numerous technical papers on the dynamics of tether in space. ${ }^{26-28}$ Some recent papers discuss the elasticity and vibration of the tethers. ${ }^{28,29}$ Compared to the early literature focused on the twobody dynamics for tether retrieval and momentum exchange purposes, recent research efforts investigate the dynamics of a three-body inline configuration ${ }^{31,35}$ and a triangular configuration. ${ }^{30,32,34}$ This paper introduces reduction of the original dynamics by oscillation synchronization, as observed in the Huygens' clocks. ${ }^{5,36}$ We pay particular attention to oscillations of the compound pendulum mode. Most of the previous work does not include this mode, by assuming that the spacecraft can be regarded as a point mass with a longer tether. However, it is indispensable to examine this mode because of the instability occurring while retracting the tether for spinning arrays, as shown in section II. Nonlinear model reduction theory of controlled Largrangian and Hamiltoniam is an area of intense research, especially in the sense of geometric control theory. ${ }^{37,38}$ Spatial reduction of linear systems with input-output symmetry can be traced back to the use of circulant matrix. ${ }^{40}$ Our approach to nonlinear model reduction is unique in the sense that we use oscillation sychronization to simplify the coupled dynamics into the simplest form whose combined stability can be analyzed systematically.

The organization of this paper is as follows: Modeling of single and double spacecraft systems is presented along with a brief introduction of contraction theory in Section II. The stability of the decentralized control law is proven in the local and global convergence sense in Section III. The results are extended to multiplespacecraft arrays in Section IV. Simulation and experimental results are detailed in Section V.

\section{Dynamics Modeling and Contraction Theory}

\section{A. Single-Tethered System}

The equations of motion of a single-tethered system in Figure 2 are presented here. The tether is assumed to be ideal- i.e. massless and inextensible; therefore, neither longitudinal nor transverse vibrations of tether are allowed. The zero mass assumption can be realized by rather strong thin material like Kevlar ${ }^{26}$ to avoid a detrimental phenomenon such as the coupling between in-plane and out-plane oscillations of the massive tether and the spacecraft attitude. ${ }^{33}$ Additionally, the array is assumed to always rotate at a certain angular rate so the tether is taut and straight at all times. A nonzero angular rotation ${ }^{32}$ is a realistic assumption since tethered interferometers will attempt to fill a full $\mathrm{u}-\mathrm{v}$ coverage $^{1}$ by rotation. For simplicity, the speed 
of the tether motor can be easily pre-defined in the control code and does not constitute the system states. This tether velocity control has been successfully validated with the SPHERES testbed, as shown in section V.

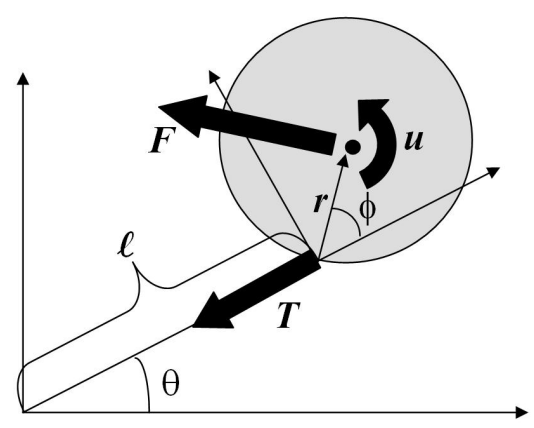

Figure 2. Free-body diagram of a revolving tether problem

The equations of motion can be derived by exploiting Lagrange's equations.

$$
\begin{gathered}
L(\mathbf{q}, \dot{\mathbf{q}})=K(\mathbf{q}, \dot{\mathbf{q}})-V(\mathbf{q})=\frac{1}{2} \dot{\mathbf{q}}^{T} \mathbf{M}(\mathbf{q}) \dot{\mathbf{q}}-V(\mathbf{q}) \\
\frac{d}{d t} \frac{\partial L}{\partial \dot{q}_{i}}-\frac{\partial L}{\partial q_{i}}=\tau_{i}
\end{gathered}
$$

The gravity term, $V(\mathbf{q})$ is omitted on the assumption that a tethered formation array such as SPECS will operate in a very weak gravity field - e.g. the second Lagrangian point L2 of the Earth-Sun system. The operation of tethered formation arrays in any kind of Earth orbit is not plausible due to expensive fuel consumption and unsatisfactory photon yield. ${ }^{30}$ Control-wise, any additional unmodeled gravity is regarded as a low-frequency disturbance to the system. In addition, out-of-plane motions are eliminated because they can be simply decoupled from the in-plane dynamics, and hence controllable by out-of-plane thruster firings. ${ }^{25}$ Then, the governing equations of motion on the array rotational plane (aperture pupil plane) become

$$
\mathbf{M}_{\mathbf{1}}(\phi)\left(\begin{array}{c}
\ddot{\theta} \\
\ddot{\phi}
\end{array}\right)+\mathbf{C}_{\mathbf{1}}(\phi, \dot{\theta}, \dot{\phi})\left(\begin{array}{c}
\dot{\theta} \\
\dot{\phi}
\end{array}\right)+\left(\begin{array}{c}
2 m(r \cos \phi+\ell) \dot{\theta} \dot{\ell} \\
2 m r \cos \phi \dot{\theta} \dot{\ell}
\end{array}\right)=\left(\begin{array}{c}
\tau_{\theta} \\
\tau_{\phi}
\end{array}\right)
$$

$$
\begin{aligned}
& \text { where } \mathbf{M}_{\mathbf{1}}(\phi)=\left[\begin{array}{cc}
m_{11}(\phi) & m_{12}(\phi) \\
m_{12}(\phi) & m_{22}
\end{array}\right]=\left[\begin{array}{cc}
I_{r}+m \ell^{2}+2 m r \ell \cos \phi & I_{r}+m r \ell \cos \phi \\
I_{r}+m r \ell \cos \phi & I_{r}
\end{array}\right], \\
& \mathbf{C}_{\mathbf{1}}(\phi, \dot{\theta}, \dot{\phi})=\left[\begin{array}{cc}
c_{11}(\phi, \dot{\phi}) & c_{12}(\phi, \dot{\theta}, \dot{\phi}) \\
c_{21}(\phi, \dot{\theta}) & c_{22}
\end{array}\right]=\left[\begin{array}{cc}
-m r \ell \sin \phi \dot{\phi} & -m r \ell \sin \phi(\dot{\theta}+\dot{\phi}) \\
+m r \ell \sin \phi \dot{\theta} & 0
\end{array}\right], \text { and } \\
& \left(\begin{array}{c}
\tau_{\theta} \\
\tau_{\phi}
\end{array}\right)=\left[\begin{array}{cc}
r+\ell \cos \phi & 1 \\
r & 1
\end{array}\right]\left(\begin{array}{l}
F \\
u
\end{array}\right) .
\end{aligned}
$$

In the equations above, $r, \ell$, and $I_{G}$ denote the satellite's radius, tether length, and moment of inertia. $I_{r}$ is the moment of inertia about the tether attachment point $\left(I_{r}=I_{G}+m r^{2}\right)$. $F$ is the linear force due to thruster firing, and $u$ is the torque exerted on the Center of Mass (CM) of the satellite, e.g. torque by a Reaction Wheel Assembly (RWA).

Note that the particular definition of the $\mathbf{C}_{\mathbf{1}}$ matrix $^{16}$ above implies that that $\left(\dot{\mathbf{M}}_{\mathbf{1}}-2 \mathbf{C}_{\mathbf{1}}\right)$ is skewsymmetric.

This system has a rigid body mode of $\theta$ and unforced natural frequency of compound pendulum mode of $\omega_{\phi}=\sqrt{\frac{r\left(I_{r}+m \ell(2 r+\ell)\right)}{l I_{G}}} \omega[\mathrm{rad} / \mathrm{s}]$ when it is linearized about a nominal $\dot{\theta}=\omega$ and $\dot{\phi}, \phi=0$. It can be shown that the system goes unstable when the tether motor reels in by checking the eigenvalues of the Linear Time-Invariant (LTI) system ${ }^{24}$

$$
\frac{d}{d t}\left(\begin{array}{c}
\theta \\
\phi \\
\dot{\theta} \\
\dot{\phi}
\end{array}\right)=\left[\begin{array}{cccc}
0 & 0 & 1 & 0 \\
0 & 0 & 0 & 1 \\
0 & \frac{r \omega^{2}\left(I_{r}+m r \ell\right)}{\ell I_{G}} & -2 \frac{\ell}{\ell} & 0 \\
0 & -\frac{r \omega^{2}\left(I_{r}+m \ell(2 r+\ell)\right)}{\ell I_{G}} & 2 \frac{\ell}{\ell} & 0
\end{array}\right]\left(\begin{array}{c}
\theta \\
\phi \\
\dot{\theta} \\
\dot{\phi}
\end{array}\right)+\left(\begin{array}{cc}
0 & 0 \\
0 & 0 \\
\frac{1}{m \ell} & -\frac{r}{I_{G} \ell} \\
-\frac{1}{m \ell} & \frac{r+\ell}{I_{G} \ell}
\end{array}\right)\left(\begin{array}{l}
F \\
u
\end{array}\right)
$$

In other words, a positive reel-out speed $(\dot{\ell}>0)$ resulted in damping of both $\dot{\theta}$ and pendulum motion of $\phi$ whereas we will see unstable motions of states for a negative reel-in speed $(\dot{\ell}<0)$. It is also easy to verify that the system with a nonzero $\omega$ is fully controllable by $F$ and $u$ using Eq. (2). ${ }^{24}$ 


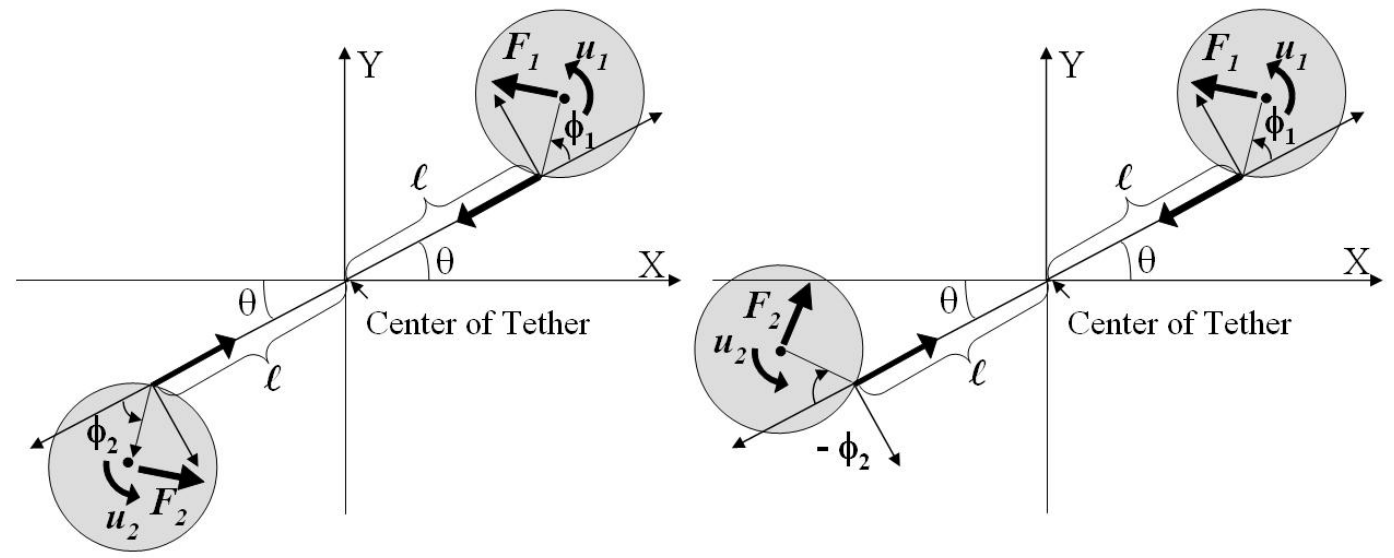

Figure 3. Free-body diagram of tethered two satellites. Synchronized $\phi$ (Left), Anti-Synchronized (Right)

\section{B. Reduction of Two Spacecraft Dynamics}

The dynamics of two tethered satellites shown on the plane of rotation in Figure 3 are derived using Lagrangian equations. As shown in the figure, the absolute position of the origin of the $\mathrm{X}-\mathrm{Y}$ axes might translate on the 2-D plane, but we will focus on the relative dynamics. Note that the origin coincides with the CM of the array when $\phi$ angles are in synchrony (in phase), as seen in Figure 3, and the CM of the array is controlled by a separate controller. The positive direction of both $\phi$ angles is defined as clockwise. The assumption of all identical spacecraft in size, mass and inertia property allows us to develop the following equations of motions:

$$
\mathbf{M}_{2}\left(\phi_{1}, \phi_{2}\right)\left(\begin{array}{c}
\ddot{\theta} \\
\ddot{\phi_{1}} \\
\ddot{\phi_{2}}
\end{array}\right)+\mathbf{C}_{2}\left(\phi_{1}, \phi_{2}, \dot{\theta}, \dot{\phi}_{1}, \dot{\phi}_{2}\right)\left(\begin{array}{c}
\dot{\theta} \\
\dot{\phi_{1}} \\
\dot{\phi_{2}}
\end{array}\right)+\left(\begin{array}{c}
2 m\left(r \cos \phi_{1}+r \cos \phi_{2}+2 \ell\right) \dot{\theta} \dot{\ell} \\
2 m r \cos \phi_{1} \dot{\theta} \dot{\ell} \\
2 m r \cos \phi_{2} \dot{\theta} \dot{\ell}
\end{array}\right)=\left(\begin{array}{c}
\tau_{\theta, 1}+\tau_{\theta, 2} \\
\tau_{\phi, 1} \\
\tau_{\phi, 2}
\end{array}\right)
$$

where $\mathbf{M}_{\mathbf{2}}=$

$$
\begin{gathered}
{\left[\begin{array}{ccc}
2 I_{r}+2 m \ell^{2}+2 m r \ell \cos \phi_{1}+2 m r \ell \cos \phi_{2} & I_{r}+m r \ell \cos \phi_{1} & I_{r}+m r \ell \cos \phi_{2} \\
I_{r}+m r \ell \cos \phi_{1} & I_{r} & 0 \\
I_{r}+m r \ell \cos \phi_{2} & 0 & I_{r}
\end{array}\right],} \\
\mathbf{C}_{\mathbf{2}}\left(\phi_{1}, \phi_{2}, \dot{\theta}, \dot{\phi_{1}}, \dot{\phi}_{2}\right)=\left[\begin{array}{ccc}
c_{11}\left(\phi_{1}, \dot{\phi}_{1}\right)+c_{11}\left(\phi_{2}, \dot{\phi}_{2}\right) & c_{12}\left(\phi_{1}, \dot{\theta}, \dot{\phi}_{1}\right) & c_{12}\left(\phi_{2}, \dot{\theta}, \dot{\phi_{2}}\right) \\
c_{21}\left(\phi_{1}, \dot{\theta}\right) & c_{22} & 0 \\
c_{21}\left(\phi_{2}, \dot{\theta}\right) & 0 & c_{22}
\end{array}\right]
\end{gathered}
$$

where $\tau_{\theta, k}, \tau_{\phi, k}$, and $c_{i j}$ are given in Eq. (1). $I_{r}$ is again defined as $I_{r}=I_{G}+m r^{2}$.

The tether length is now $2 \ell$ and $\dot{\ell}$ is half the speed of the tether. The array angular rate $\dot{\theta}$ is assumed to be the same for both satellites. This is especially true when the tether is in tension. Furthermore, the mass and inertia properties are assumed to be roughly the same. These geometric symmetry properties are pervasive in stellar interferometers where sub-telescopes must be identical for interferometric beam combining. This Lagrangian system is also kinetic symmetric with respect to $\theta$ since $\frac{\partial K}{\partial \theta}=0$. In fact, the kinetic symmetry leads to the symmetry in mechanics ${ }^{37}$ in the absence of a potential field, i.e. $\frac{\partial L}{\partial \theta}=0$, which in turn corresponds to the conservation of the momentum $\left(\frac{\partial L}{\partial \dot{\theta}}=\right.$ constant $)$ for an unforced system. Note that both the single and two spacecraft systems are symmetric in mechanics with respect to $\theta$, thereby resulting an inertia matrix independent of $\theta$. This independent variable is often called external variable. ${ }^{22}$

It can be inferred by inspecting Eq. (3) that the first row of the equation is the only coupled term of $\phi_{1}$ and $\phi_{2}$, which is the sum of the first rows of two independent single-tethered systems of the form in Eq. (1): 


$$
\begin{aligned}
& \mathbf{M}_{\mathbf{1}}\left(\phi_{1}\right)\left(\begin{array}{c}
\ddot{\theta} \\
\ddot{\phi_{1}}
\end{array}\right)+\mathbf{C}_{\mathbf{1}}\left(\phi_{1}, \dot{\theta}, \dot{\phi}_{1}\right)\left(\begin{array}{c}
\dot{\theta} \\
\dot{\phi_{1}}
\end{array}\right)+\left(\begin{array}{c}
2 m\left(r \cos \phi_{1}+\ell\right) \dot{\theta} \dot{\ell} \\
2 m r \cos \phi_{1} \dot{\theta} \dot{\ell}
\end{array}\right)=\left(\begin{array}{c}
\tau_{\theta, 1} \\
\tau_{\phi, 1}
\end{array}\right)=\tau_{\mathbf{1}} \\
& \mathbf{M}_{\mathbf{1}}\left(\phi_{2}\right)\left(\begin{array}{c}
\ddot{\theta} \\
\ddot{\phi_{2}}
\end{array}\right)+\mathbf{C}_{\mathbf{1}}\left(\phi_{2}, \dot{\theta}, \dot{\phi}_{2}\right)\left(\begin{array}{c}
\dot{\theta} \\
\dot{\phi_{2}}
\end{array}\right)+\left(\begin{array}{c}
2 m\left(r \cos \phi_{2}+\ell\right) \dot{\theta} \dot{\ell} \\
2 m r \cos \phi_{2} \dot{\theta} \dot{\ell}
\end{array}\right)=\left(\begin{array}{c}
\tau_{\theta, 2} \\
\tau_{\phi, 2}
\end{array}\right)=\tau_{\mathbf{2}}
\end{aligned}
$$

where $\mathbf{M}_{\mathbf{1}}(\phi), \mathbf{C}_{\mathbf{1}}(\phi, \dot{\theta}, \dot{\phi})$ are given in Eq. (1).

It is easily verified that Eq. (3) reduces to Eqs. (4-5) if $\phi_{1}$ and $\phi_{2}$ can somehow be driven to oscillate in synchrony. In other words, we can decouple the dynamics of the first satellite from the other resulting in the same equation as Eq. (1) if the controller $u_{1}$ and $u_{2}$ make $\phi_{1}$ and $\phi_{2}$ converge towards each other exponentially fast. This important finding leads to the following theorem.

\section{Definition 1: Decentralized Control Law}

Assume that for a two-spacecraft tethered system, some control function $\mathbf{u}$ is decentralized, in the sense that it does not require state information from the other satellite, ${ }^{12}$ and that

$$
\tau_{\mathbf{1}}=\mathbf{u}\left(\phi_{1}, \dot{\theta}, \dot{\phi}_{1}\right) \text { and } \tau_{\mathbf{2}}=\mathbf{u}\left(\phi_{2}, \dot{\theta}, \dot{\phi}_{2}\right) \text { where } \mathbf{u} \text { is the same control function }
$$

Assume further that the strictly stabilizing control laws, $\tau_{\mathbf{1}}$ and $\tau_{\mathbf{2}}$, designed based on the single tethered systems in Eq. (4) and (5) respectively, also strictly stabilize the combined system in Eq. (3). Then a decentralized control law, $\tau_{\mathbf{i}}=u\left(\phi_{i}, \dot{\theta}, \dot{\phi}_{i}\right), i=1,2$ can be designed from a single-tethered system given in Eqs. (4-5) in lieu of the two body dynamics in Eq. (3). In addition, this control law u makes $\phi_{1}$ and $\phi_{2}$ converge to each other.

This decentralized control law will significantly simplify both the control algorithm and satellite hardware with few complications in terms of dimensionality and no communication burden. Basically, the fixed center of the rotation for a single-tethered system will be replaced with the center of the tether of the two satellites (see figure 3). Subsequent sections demonstrate the existence of such controller as in Definition 1 in two ways: locally by linear diagonalizing transformation (Section III-A) and globally by contraction theory (Section III-B).

\section{Review of Contraction Theory}

Lyapunov's linearization method indicates that the local stability of the nonlinear system can be analyzed using its differential approximation. What is new in contraction theory is that a differential stability analysis can be made exact, thereby yielding global results on the nonlinear system. A brief review of the results from Ref. 17-20 is presented in this section. Readers are referred to these references for detailed descriptions and proofs on the following theorems.

A nonlinear system, possibly a time varying non-autonomous system is formulated as:

$$
\dot{\mathbf{x}}=\mathbf{f}(\mathbf{x}, \mathbf{u}(\mathbf{x}, t), t)
$$

A virtual displacement, $\delta \mathbf{x}$ is defined as an infinitesimal displacement at fixed time, which is commonly found in the calculus of variations.

Theorem 1: For the system in Eq. (6), if there exists a uniformly positive definite metric,

$$
\mathbf{M}(\mathbf{x}, t)=\Theta(\mathbf{x}, t)^{T} \Theta(\mathbf{x}, t)
$$

where $\Theta$ is some smooth coordinate transformation of the virtual displacement, $\delta \mathbf{z}=\Theta \delta \mathbf{x}$, such that the associated generalized Jacobian, $\mathbf{J}$ is uniformly negative definite, i.e., $\exists \lambda>0$ such that

$$
\mathbf{J}=\left(\dot{\Theta}(\mathbf{x}, t)+\Theta(\mathbf{x}, t) \frac{\partial \mathbf{f}}{\partial \mathbf{x}}\right) \Theta(\mathbf{x}, t)^{-1} \leq-\lambda \mathbf{I},
$$

then all system trajectories converge globally to a single trajectory exponentially fast regardless of the initial conditions, with a global exponential convergence rate of the largest eigenvalues of the symmetric part of $\mathbf{J}$. 
Such system is said to be contracting. The proof is given in Ref. 17. Equivalently, the system is contracting if $\exists \lambda>0$ such that

$$
\dot{\mathbf{M}}+\left(\frac{\partial \mathbf{f}}{\partial \mathbf{x}}\right)^{T} \mathbf{M}+\mathbf{M} \frac{\partial \mathbf{f}}{\partial \mathbf{x}} \leq-2 \lambda \mathbf{M}
$$

It can also be shown that for a contracting autonomous system, of the form $\dot{\mathbf{x}}=\mathbf{f}(\mathbf{x}, \mathbf{u}(\mathbf{x}))$, all trajectories converges to an equilibrium point exponentially fast. In essence, contraction analysis implies that stability of nonlinear systems can be analyzed more simply by checking the negative definiteness of a proper matrix, rather than finding some implicit motion integral as in Lyapunov theory. Note that contraction theory is a generalization of the classical Krasovskii's theorem. ${ }^{16}$

The following theorems are used to derive stability of the coupled dynamics systems.

Theorem 2: Hierarchical Combination ${ }^{18,19}$ Consider two contracting systems, of possibly different dimensions and metrics, and connect them in series, leading to a smooth virtual dynamics of the form

$$
\frac{d}{d t}\left(\begin{array}{l}
\delta \mathbf{z}_{1} \\
\delta \mathbf{z}_{2}
\end{array}\right)=\left(\begin{array}{cc}
\mathbf{J}_{11} & \mathbf{0} \\
\mathbf{J}_{21} & \mathbf{J}_{22}
\end{array}\right)\left(\begin{array}{l}
\delta \mathbf{z}_{1} \\
\delta \mathbf{z}_{2}
\end{array}\right)
$$

Then the combined system is contracting if $\mathbf{J}_{\mathbf{2 1}}$ is bounded.

Theorem 3: Synchronization and Partial Contraction ${ }^{19}$ Consider two coupled systems. If the dynamics equations verify

$$
\dot{\mathbf{x}}_{1}-\mathbf{f}\left(\mathbf{x}_{1}, t\right)=\dot{\mathbf{x}}_{2}-\mathbf{f}\left(\mathbf{x}_{2}, t\right)
$$

where the function $\mathbf{f}(\mathbf{x}, t)$ is contracting in an input-independent metric, then $\mathbf{x}_{1}$ and $\mathbf{x}_{2}$ will converge to each other exponentially, regardless of the initial conditions.

\section{Stability of Decentralized Control Law}

\section{A. Diagonalization of Linearized System}

The two-spacecraft equation (3) with the tether length fixed $(\dot{\ell}=0)$ can also be linearized about $\dot{\theta}=\omega$, and $\dot{\phi}, \phi=0$ with $F_{1}=0, F_{2}=0$ (torque actuation only):

$$
\left[\begin{array}{ccc}
2 I_{r}+2 m \ell(2 r+\ell) & I_{r}+m r \ell & I_{r}+m r \ell \\
I_{r}+m r \ell & I_{r} & 0 \\
I_{r}+m r \ell & 0 & I_{r}
\end{array}\right]\left(\begin{array}{c}
\ddot{\theta} \\
\ddot{\phi_{1}} \\
\ddot{\phi_{2}}
\end{array}\right)+\left[\begin{array}{ccc}
0 & 0 & 0 \\
0 & m r \ell \omega^{2} & 0 \\
0 & 0 & m r \ell \omega^{2}
\end{array}\right]\left(\begin{array}{c}
\theta \\
\phi_{1} \\
\phi_{2}
\end{array}\right)=\left(\begin{array}{c}
u_{1}+u_{2} \\
u_{1} \\
u_{2}
\end{array}\right)
$$

It is observed that the nonzero rotational rate, $\omega$, added a potential term to the dynamics even though there is no gravitational force in the model. This nonzero artificial potential energy induced by the centrifugal force of array rotation, plays a crucial role in making the system controllable and stable. ${ }^{24}$ This is especially true for large classes of underactuated systems (e.g. tethered systems with $F=0$ ), which are neither controllable in the absence of potential energy nor fully feedback linearizable. ${ }^{22}$

The natural frequencies of this LTI system are computed as $\omega_{\theta}=0, \omega_{\phi}=\sqrt{\frac{r\left(I_{r}+m \ell(2 r+\ell)\right)}{l I_{G}}} \omega$, and $\omega_{o}=\sqrt{\frac{m r \ell}{I_{r}}} \omega$. Note that $\omega_{\phi}$ is the same compound pendulum mode frequency as in the single spacecraft case, and $\omega_{\theta}$ represents the same rigid body mode of $\theta$ and $\dot{\theta}$ as in the single-tethered system, whereas $\omega_{o}$ is the natural frequency of the anti-synchronization (out-of-phase) mode depicted in Figure 3. It can be shown that $\omega_{o}$ is always smaller than $\omega_{\phi}$.

We can diagonalize the system in Eq. (10) using normalized eigenvectors. ${ }^{24}$ Then, with a linear decentralized control law of the form:

$$
u_{i}=-K_{1} \phi_{i}-K_{2} \dot{\theta}-K_{3} \dot{\phi}_{i} i=1,2
$$

equation (10) can be diagonalized as 


$$
\begin{gathered}
\ddot{z}_{1}=s_{1} \dot{z}_{1}+s_{2} \dot{z}_{2}+p_{1} z_{2} \\
\ddot{z}_{2}+\omega_{\phi}^{2} z_{2}=s_{3} \dot{z}_{1}+s_{4} \dot{z}_{2}+p_{2} z_{2} \\
\ddot{z}_{3}+\frac{K_{3}}{I_{r}} \dot{z}_{3}+\left(\omega_{o}^{2}+\frac{K_{1}}{I_{r}}\right) z_{3}=0
\end{gathered}
$$

where $s_{1}=\frac{-K_{2}}{I_{r}+m \ell(2 r+\ell)}, s_{2}=\frac{I_{G}\left(K_{2}-K_{3}\right)+m(r+\ell)\left(r K_{2}-K_{3}(r+\ell)\right)}{\sqrt{I_{G} \ell^{2} m\left(I_{r}+m \ell(2 r+\ell)\right)}}, s_{3}=\frac{m \ell(r+\ell)}{\sqrt{I_{G} \ell^{2} m}} q_{1}, s_{4}=(r+\ell) \sqrt{\frac{m}{I_{G}}} q_{2}, p_{1}=$ $\frac{-K_{1}}{\sqrt{I_{G} \ell^{2} m}}$, and $p_{2}=\frac{-K_{1}(r+\ell)}{I_{G} \ell}$

By inspecting the eigenvectors given in Ref. 24, it is clear that $z_{1}$ represents $\theta$ and $z_{2}$ is the dynamics of the synchronized compound pendulum mode $\left(\phi_{1}+\phi_{2}\right)$ in figure 3 with the same natural frequency $\omega_{\phi}$ as in the single-tethered case. The additional mode is the compound pendulum mode of anti-synchrony, which is $z_{3} \propto \phi_{1}-\phi_{2}$.

Likewise, diagonalizing the linearized single spacecraft system in Eq.(1) yields the same normalized equations of motions for $z_{1}$ and $z_{2}$ in Eq. (12). Thus, the stabilizing controller $u_{i}$ from the single-tethered system will stabilize $\theta$ and the synchronized compound pendulum mode $z_{2}$. Now satisfying Definition 1 comes down to finding conditions in which the anti-synchronized mode $z_{3}$ is stabilized.

The global stability condition of $K_{1}$ and $K_{3}$ for the linear $z_{3}$ dynamics in Eq. (12) can be found as $K_{3}>0$ and $\omega_{o}^{2}+\frac{K_{1}}{I_{r}}>0$.

Similarly, it can be shown that any controller satisfying $r K_{2}<(r+\ell) K_{3}, K_{1}>0, K_{2}>0$ can stabilize $z_{1}$ and $z_{2}$. Note that $K_{3}>0$ and $\omega_{o}^{2}+\frac{K_{1}}{I_{r}}>0$ are automatically satisfied by this condition, thereby stabilizing the coupled two-body system in Eq. (10). This proves the existence of such controller as in Definition 1.

Theorem 4: Effect of nonzero speed of the tether $(\dot{\ell} \neq 0)$

When the tether motors reel in or out, $\dot{\ell}$ is a nonzero term. It can be shown that $\dot{\ell}$ does not affect the anti-synchronizing mode $z_{3}$; therefore a controller stabilizing a single-tethered system with a constant $\dot{\ell} \neq 0$, can be used to stabilize a two-spacecraft system.

\section{B. Nonlinear Model Reduction by Contraction Theory}

The stability of the anti-synchronizing compound pendulum mode $z_{3}$ in Eq. (12) can be verified more easily using contraction theory, specifically Theorems 2 and 3. From the second and third row of Eq. (10),

$$
\begin{aligned}
& \left(I_{r}+m r \ell\right) \ddot{\theta}+I_{r} \ddot{\phi}_{1}+m r \ell \omega^{2} \phi_{1}=-K_{1} \phi_{1}-K_{3} \dot{\phi}_{1}-K_{2} \dot{\theta} \\
& \left(I_{r}+m r \ell\right) \ddot{\theta}+I_{r} \ddot{\phi}_{2}+m r \ell \omega^{2} \phi_{2}=-K_{1} \phi_{2}-K_{3} \dot{\phi}_{2}-K_{2} \dot{\theta}
\end{aligned}
$$

Subtracting the first line from the second in Eq. (13) results in:

$$
I_{r} \ddot{\phi_{1}}+m r \ell \omega^{2} \phi_{1}+K_{1} \phi_{1}+K_{3} \dot{\phi_{1}}=I_{r} \ddot{\phi_{2}}+m r l \omega^{2} \phi_{2}+K_{1} \phi_{2}+K_{3} \dot{\phi_{2}}
$$

According to Theorem 3, if the $z_{3}$ dynamics in Eq. (12) are exponentially stable, then $\phi_{1}$ converges to $\phi_{2}$ exponentially fast. Then, the stability of the original system depends on the stability of the reduced singletethered system. Furthermore, $\left(I_{r}+m r \ell\right) \ddot{\theta}$ can be regarded as an auxiliary input to the partially contracting dynamics of $z_{3}$. Therefore, a stable closed-loop single-tethered dynamics stabilizes the $z_{3}$ dynamics.

The previous analysis is limited to the linear Proportional and Derivative (PD) controller given in Eq. (11). This is a local stability result based on the linearized dynamics. On the other hand, contraction theory exploits a differential analysis to derive a global stability result for the nonlinear dynamics. In addition, the simple PD control cannot be expected to handle the dynamic demands of trajectory tracking efficiently. ${ }^{16}$ Thus, an exponentially stable nonlinear controller is proposed, and contraction analysis is used to check whether the combination of exponentially stable single-tethered systems leads to exponential stability.

Consider a single-tethered system in Eq. (4) with the tether length fixed $(\dot{\ell}=0)$,

$$
\mathbf{M}_{1}\left(\mathbf{q}_{1}\right) \ddot{\mathbf{q}}_{1}+\mathbf{C}_{1}\left(\mathbf{q}_{1}, \dot{\mathbf{q}}_{1}\right) \dot{\mathbf{q}}_{1}=\tau_{1}
$$

where $\mathbf{M}_{\mathbf{1}}, \mathbf{C}_{\mathbf{1}}$, and $\tau_{\mathbf{1}}$ are given in Eq. (1). In addition, $\mathbf{q}_{\mathbf{1}}$ is defined as $\left(\begin{array}{ll}\theta & \phi_{1}\end{array}\right)^{T}$. 
The following tracking controller is proposed in Ref. 21:

$$
\tau_{1}=\mathbf{M}_{1}\left(\mathbf{q}_{1}\right) \ddot{\mathbf{q}}_{1 \mathbf{r}}+\mathbf{C}_{\mathbf{1}}\left(\mathbf{q}_{1}, \dot{\mathbf{q}}_{1}\right) \dot{\mathbf{q}}_{1 \mathbf{r}}-\mathbf{K}\left(\dot{\mathbf{q}}_{1}-\dot{\mathbf{q}}_{1 \mathbf{r}}\right)
$$

The reference velocity vector, $\dot{\mathbf{q}}_{1 \mathbf{r}}$ is given by shifting the desired velocity $\dot{\mathbf{q}}_{\mathbf{1 d}}$ with the position error:

$$
\dot{\mathbf{q}}_{1 \mathrm{r}}=\dot{\mathbf{q}}_{1 \mathrm{~d}}-\Lambda \widetilde{\mathbf{q}}_{1}=\dot{\mathbf{q}}_{1 \mathrm{~d}}-\Lambda\left(\mathbf{q}_{1}-\mathbf{q}_{1 \mathrm{~d}}\right)
$$

where $\boldsymbol{\Lambda}$ is a symmetric positive definite matrix.

From Eq. (14) and (15), consider the following virtual system,

$$
\mathbf{M}_{1}\left(\mathbf{q}_{1}\right) \dot{\mathbf{y}}+\mathbf{C}_{1}\left(\mathbf{q}_{1}, \dot{\mathbf{q}}_{1}\right) \mathbf{y}-\mathbf{K}\left(\dot{\mathbf{q}}_{1}-\mathbf{y}\right)=\tau_{\mathbf{1}}
$$

$\dot{\mathbf{q}}_{1}$ and $\dot{\mathbf{q}}_{1 \mathbf{r}}$ are two particular solutions of this virtual dynamics, which is obvious by substituting for $\mathbf{y}$.

For system (17), we select the inertia matrix $\mathbf{M}_{\mathbf{1}}\left(\mathbf{q}_{\mathbf{1}}\right)$ as a metric in Eq. (7), which is uniformly positive definite. Then, the squared length of the corresponding $\delta \mathbf{z}$ can be written $\delta \mathbf{z}^{T} \delta \mathbf{z}=\delta \mathbf{y}^{T} \mathbf{M}_{\mathbf{1}}\left(\mathbf{q}_{\mathbf{1}}\right) \delta \mathbf{y}$, and its derivative can be computed directly from (17),

$$
\begin{aligned}
& \frac{d}{d t}\left(\delta \mathbf{y}^{T} \mathbf{M}_{\mathbf{1}}\left(\mathbf{q}_{\mathbf{1}}\right) \delta \mathbf{y}\right)=2 \delta \mathbf{y}^{T} \mathbf{M}_{\mathbf{1}}\left(\mathbf{q}_{\mathbf{1}}\right) \delta \dot{\mathbf{y}}+\delta \mathbf{y}^{T} \dot{\mathbf{M}}_{\mathbf{1}}\left(\mathbf{q}_{\mathbf{1}}\right) \delta \mathbf{y} \\
& \quad=-2 \delta \mathbf{y}^{T}\left(\mathbf{C}_{\mathbf{1}}\left(\mathbf{q}_{\mathbf{1}}, \dot{\mathbf{q}}_{\mathbf{1}}\right) \delta \mathbf{y}+\mathbf{K} \delta \mathbf{y}\right)+\delta \mathbf{y}^{T} \dot{\mathbf{M}}_{\mathbf{1}}\left(\mathbf{q}_{\mathbf{1}}\right) \delta \mathbf{y}=-2 \delta \mathbf{y}^{T} \mathbf{K} \delta \mathbf{y}
\end{aligned}
$$

where we used the skew symmetric property, $\mathbf{y}^{T}\left(\dot{\mathbf{M}}_{\mathbf{1}}-2 \mathbf{C}_{\mathbf{1}}\right) \mathbf{y}=0$.

The virtual system of $\mathbf{y}$ is shown to be contracting with a uniformly positive definite $\mathbf{K}$ with respect to $\theta$ and $\phi_{1}$ using contraction analysis in section II. Then, the two particular solutions, $\dot{\mathbf{q}}_{1}$ and $\dot{\mathbf{q}}_{1 \mathbf{r}}$ converge to each other exponentially fast using Theorem 3.

Eq. (16) implies that $\mathbf{q}_{\mathbf{1}} \rightarrow \mathbf{q}_{\mathbf{1} \mathbf{d}}$ exponentially fast according to the hierarchical combination of contracting systems (Theorem 2)

$$
\dot{\tilde{\mathbf{q}}}_{1}=\left(\dot{\mathbf{q}}_{1}-\dot{\mathbf{q}}_{1 \mathbf{r}}\right)-\Lambda \widetilde{\mathbf{q}}_{1}
$$

One choice of $\mathbf{K}$ is

$$
\mathbf{K}=\left[\begin{array}{ll}
k_{11} & k_{12} \\
k_{12} & k_{22}
\end{array}\right] \quad \text { where } \quad k_{11}>0 \text { and } k_{22}-\frac{k_{12}^{2}}{k_{11}}>0
$$

Similarly, we can construct an exponentially stabilizing tracking controller for the second spacecraft with $\mathbf{q}_{2}=\left(\begin{array}{ll}\theta & \phi_{2}\end{array}\right)^{T}$.

$$
\tau_{2}=\mathbf{M}_{1}\left(\mathbf{q}_{2}\right) \ddot{\mathbf{q}}_{2 \mathbf{r}}+\mathbf{C}_{1}\left(\mathbf{q}_{2}, \dot{\mathbf{q}}_{2}\right) \dot{\mathbf{q}}_{2 \mathbf{r}}-\mathbf{K}\left(\dot{\mathbf{q}}_{2}-\dot{\mathbf{q}}_{2 \mathbf{r}}\right)
$$

This controller can be shown to exponentially stabilize the second spacecraft dynamics in Eq.(5) with the same $\mathbf{K}$ as in Eq. (19).

Let us now check whether these controllers from the single-tethered systems can exponentially stabilize the two-spacecraft tethered system in Eq. (3).

For the first spacecraft given in Eq. (14), we augment the states, $\theta, \phi$ with an auxiliary coordinate $\phi_{2}$ such that

$$
\begin{gathered}
\mathbf{M}_{\phi_{1}}(\mathbf{q}) \ddot{\mathbf{q}}+\mathbf{C}_{\phi_{1}}(\mathbf{q}, \dot{\mathbf{q}}) \dot{\mathbf{q}}=\tau_{\mathbf{a}} \\
\tau_{\mathbf{a}}=\mathbf{M}_{\phi_{1}}(\mathbf{q}) \ddot{\mathbf{q}}_{\mathbf{r}}+\mathbf{C}_{\phi_{1}}(\mathbf{q}, \dot{\mathbf{q}}) \dot{\mathbf{q}}_{\mathbf{r}}-\mathbf{K}_{\mathbf{D} 1}\left(\dot{\mathbf{q}}-\dot{\mathbf{q}}_{\mathbf{r}}\right)
\end{gathered}
$$

where

$\mathbf{M}_{\phi_{\mathbf{1}}}=\left[\begin{array}{ccc}m_{11} & m_{12} & 0 \\ m_{12} & m_{22} & 0 \\ 0 & 0 & 0\end{array}\right]_{\phi=\phi_{1}}, \mathbf{C}_{\phi_{\mathbf{1}}}=\left[\begin{array}{ccc}c_{11} & c_{12} & 0 \\ c_{21} & c_{22} & 0 \\ 0 & 0 & 0\end{array}\right]_{\substack{\phi=\phi_{1} \\ \dot{\phi}=\dot{\phi}_{1}}}, \tau_{\mathbf{a}}=\left(\begin{array}{c}\tau_{\theta, 1} \\ \tau_{\phi, 1} \\ 0\end{array}\right), \mathbf{K}_{\mathbf{D} \mathbf{1}}=\left[\begin{array}{ccc}k_{11} & k_{12} & 0 \\ k_{12} & k_{22} & 0 \\ 0 & 0 & 0\end{array}\right]$, and $\mathbf{q}=\left(\begin{array}{c}\theta \\ \phi_{1} \\ \phi_{2}\end{array}\right)$

Equation (21) is the equation of motion of $\theta$ and $\phi_{1} ; \phi_{2}$ is added just as a dummy variable to facilitate the proof in this section. 
Similarly, we can augment the dynamics equations of the second spacecraft as

$$
\begin{gathered}
\mathbf{M}_{\phi_{\mathbf{2}}}(\mathbf{q}) \ddot{\mathbf{q}}+\mathbf{C}_{\phi_{\mathbf{2}}}(\mathbf{q}, \dot{\mathbf{q}}) \dot{\mathbf{q}}=\tau_{\mathbf{b}} \\
\tau_{\mathbf{b}}=\mathbf{M}_{\phi_{\mathbf{2}}}(\mathbf{q}) \ddot{\mathbf{q}}_{\mathbf{r}}+\mathbf{C}_{\phi_{\mathbf{2}}}(\mathbf{q}, \dot{\mathbf{q}}) \dot{\mathbf{q}}_{\mathbf{r}}-\mathbf{K}_{\mathbf{D} \mathbf{2}}\left(\dot{\mathbf{q}}-\dot{\mathbf{q}}_{\mathbf{r}}\right)
\end{gathered}
$$

where

$$
\mathbf{M}_{\phi_{\mathbf{2}}}=\left[\begin{array}{ccc}
m_{11} & 0 & m_{12} \\
0 & 0 & 0 \\
m_{12} & 0 & m_{22}
\end{array}\right]_{\phi=\phi_{2}}, \mathbf{C}_{\phi_{\mathbf{2}}}=\left[\begin{array}{ccc}
c_{11} & 0 & c_{12} \\
0 & 0 & 0 \\
c_{21} & 0 & c_{22}
\end{array}\right]_{\substack{\phi=\phi_{2} \\
\dot{\phi}=\dot{\phi}_{2}}}, \tau_{\mathbf{b}}=\left(\begin{array}{c}
\tau_{\theta, 2} \\
0 \\
\tau_{\phi, 2}
\end{array}\right) \text {, and } \quad \mathbf{K}_{\mathbf{D} \mathbf{2}}=\left[\begin{array}{ccc}
k_{11} & 0 & k_{12} \\
0 & 0 & 0 \\
k_{12} & 0 & k_{22}
\end{array}\right]
$$

Since $\tau$ in Eq. (3) is the sum of two tracking control laws individually designed from the single-tethered systems, $\tau=\tau_{\mathbf{a}}+\tau_{\mathbf{b}}$ in Eq. (22) and (24), the virtual dynamics of the two spacecraft system becomes,

$$
\mathbf{M}_{\mathbf{2}}(\mathbf{q}) \dot{\mathbf{y}}+\mathbf{C}_{\mathbf{2}}(\mathbf{q}, \dot{\mathbf{q}}) \mathbf{y}-\left(\mathbf{K}_{\mathbf{D} \mathbf{1}}+\mathbf{K}_{\mathbf{D} \mathbf{2}}\right)(\dot{\mathbf{q}}-\mathbf{y})=\tau,
$$

which still has two particular solutions, $\dot{\mathbf{q}}$ and $\dot{\mathbf{q}}_{\mathbf{r}}$. The symmetry property discussed in Section II-B enforces $\mathbf{M}_{2}=\mathbf{M}_{\phi_{1}}+\mathbf{M}_{\phi_{2}}$ and $\mathbf{C}_{2}=\mathbf{C}_{\phi_{1}}+\mathbf{C}_{\phi_{2}}$.

The same squared-length analysis as earlier, now with respect to the uniformly positive definite metric $\mathbf{M}_{2}(\mathbf{q})$, results in

$$
\frac{d}{d t}\left(\delta \mathbf{y}^{T} \mathbf{M}_{\mathbf{2}}(\mathbf{q}) \delta \mathbf{y}\right)=-2 \delta \mathbf{y}^{T}\left(\mathbf{K}_{\mathbf{D} \mathbf{1}}+\mathbf{K}_{\mathbf{D} \mathbf{2}}\right) \delta \mathbf{y}
$$

This system is contracting, since $\mathbf{K}_{\mathbf{D} \mathbf{1}}+\mathbf{K}_{\mathbf{D} \mathbf{2}}=\left[\begin{array}{ccc}2 k_{11} & k_{12} & k_{12} \\ k_{12} & k_{22} & 0 \\ k_{12} & 0 & k_{22}\end{array}\right]$ is uniformly positive definite. This proves the existence of such controller as defined in Definition $\mathbf{1}$ for exponentially stabilizing nonlinear controllers. The global convergence of contraction analysis strengthens the local convergence result of the linear approximation of the previous section.

The result in this section can be easily extended to construction of nonlinear robust sliding controllers ${ }^{16}$ to account for tether slack dynamics and modeling uncertainties, including lack of modelling of external disturbances. In particular, slackness of the tether affects the inertia matrix $\mathbf{M}$ and specifically the $\mathbf{C}$ matrix, removing the coupling terms because the energy transfer along the tether line is instantaneously disrupted. It can be shown that a sliding controller designed from the single-tethered system will exponentially stabilize the combined system using the previous analysis and the triangle inequality.

\section{Multiple Tether Arrays}

Let us now discuss some further extensions.

\section{A. Spinning Triangular Array and Multiple Circular Array}

Motivated by successful decoupling for the two-spacecraft case, three imaginary pseudo-tethers ${ }^{25}$ connecting each satellite to the $\mathrm{CM}$ of the array are assumed to exist, replacing the three actual tether lines (see Figure 4). When the tethers are taut and straight in a rotating array, a small $\phi$ (angle of the compound pendulum mode) is approximated as a perturbed angle that the satellites make with respect to the corresponding pseudo-tether. Here, $\ell$ is defined as the length of the pseudo-tether; the actual tether length is then $L=\sqrt{3}(\ell+r)-2 r$. The original six degrees of freedom ( $\phi$ and $\theta$ for each satellite) are reduced to four with addition of two constraints, $L=\sqrt{\left(x_{i a}-x_{j a}\right)^{2}+\left(y_{i a}-y_{j a}\right)^{2}}$ with $(i, j)=(1,3),(1,2)$ where $x_{i a}=\ell \cos \theta_{i}+2 r \sin 15^{\circ} \cos \left(\theta_{i}-75^{\circ}+\phi_{i}\right)$ and $x_{j a}=\ell \cos \theta_{j}+2 r \sin 15^{\circ} \cos \left(\theta_{i}+75^{\circ}+\phi_{j}\right)$ are $x$ coordinates of tether attachment points, and $y_{i a}$ and $y_{j a}$ are similarly defined. The equations of motions of a three-spacecraft triangular array approximated by the pseudo-tethers are given in the following equation by setting $n=3$,

$$
\mathbf{M}_{\mathbf{n}}(\mathbf{q})\left(\begin{array}{c}
\ddot{\theta} \\
\ddot{\phi_{1}} \\
\ddot{\phi}_{2} \\
\vdots \\
\ddot{\phi_{n}}
\end{array}\right)+\mathbf{C}_{\mathbf{n}}\left(\begin{array}{c}
\dot{\theta} \\
\dot{\phi}_{1} \\
\dot{\phi}_{2} \\
\vdots \\
\dot{\phi}_{n}
\end{array}\right)+\left(\begin{array}{c}
2 m\left(\sum_{k=1}^{n} r \cos \phi_{k}+\ell\right) \dot{\theta} \dot{\ell} \\
2 m r \cos \phi_{1} \dot{\theta} \dot{\ell} \\
2 m r \cos \phi_{2} \dot{\theta} \dot{\ell} \\
\vdots \\
2 m r \cos \phi_{n} \dot{\theta} \dot{\ell}
\end{array}\right)=\left(\begin{array}{c}
\sum_{k=1}^{n} \tau_{\theta, k} \\
\tau_{\phi, 1} \\
\tau_{\phi, 2} \\
\vdots \\
\tau_{\phi, n}
\end{array}\right)
$$




$$
\begin{gathered}
\text { where } \mathbf{M}_{\mathbf{n}}=\left[\begin{array}{ccccc}
\sum_{k=1}^{n} m_{11}\left(\phi_{k}\right) & m_{12}\left(\phi_{1}\right) & m_{12}\left(\phi_{2}\right) & \ldots & m_{12}\left(\phi_{n}\right) \\
m_{12}\left(\phi_{1}\right) & m_{22} & 0 & \ldots & 0 \\
m_{12}\left(\phi_{2}\right) & 0 & m_{22} & \ldots & 0 \\
\vdots & 0 & 0 & \ddots & 0 \\
m_{12}\left(\phi_{n}\right) & 0 & 0 & 0 & m_{22}
\end{array}\right], \\
\mathbf{C}_{\mathbf{n}}=\left[\begin{array}{ccccc}
\sum_{k=1}^{n} c_{11}\left(\phi_{k}, \dot{\phi}_{k}\right) & c_{12}\left(\phi_{1}, \dot{\theta}, \dot{\phi_{1}}\right) & c_{12}\left(\phi_{2}, \dot{\theta}, \dot{\phi_{2}}\right) & \ldots & c_{12}\left(\phi_{n}, \dot{\theta}, \dot{\phi_{n}}\right) \\
c_{21}\left(\phi_{1}, \dot{\theta}\right) & c_{22} & 0 & \ldots & 0 \\
c_{21}\left(\phi_{2}, \dot{\theta}\right) & 0 & c_{22} & \ldots & 0 \\
\vdots & 0 & 0 & \ddots & 0 \\
c_{21}\left(\phi_{n}, \dot{\theta}\right) & 0 & 0 & 0 & c_{22}
\end{array}\right]
\end{gathered}
$$

It is straightforward to show that a stabilizing controller for a single-tethered spacecraft will stabilize a three-spacecraft triangular array using the same methods as in section III. When linearized, the system has four eigenvalues, two of which are anti-synchrony mode. Eigenvalues for the triangular array $(n=3)$ are 0 , $\sqrt{\frac{r\left(I_{r}+m \ell(2 r+\ell)\right)}{l I_{G}}} \omega, \sqrt{\frac{m r \ell}{I_{r}}} \omega$, and $\sqrt{\frac{m r \ell}{I_{r}}} \omega$.

Equation (26) originally represents the dynamics of star arrays as shown in the second row of Figure 4. Hence, the same controller designed from the singled tethered system will stabilize a star network. This method can be extended to an arbitrary number of circularly connected arrays if the dynamics equation can be represented as the form in Eq. (26). This is automatically true for $n=3$. However, we need more constraints for Eq. (26) to approximate a rotating circular array of $n \geq 4$. For spinning tethered arrays, a separate controller can control the diagonal distances to keep the pseudo tether length $\ell$ the same. In the absence of such controllers, we can add more diagonal tethers, as seen in the hexagonal array in Figure 4. The number of additional tethers required is $3 n-n-2$ (=the number of degrees-of-freedom)- $(n+1)$. In summary, under the reasonable assumption of the taut and equal-length pseudo-tether, a controller designed from a single-tethered system will synchronize both array spin rate and oscillations of each spacecraft, thereby reducing the original dynamics to those of a single-tethered spacecraft, as seen in Figure 4. The result in this section was predicted in Ref. ${ }^{19}$, in the sense that for a star network with arbitrarily many satellites, the minimum coupling strength to guarantee synchronization is independent of the number of satellites.

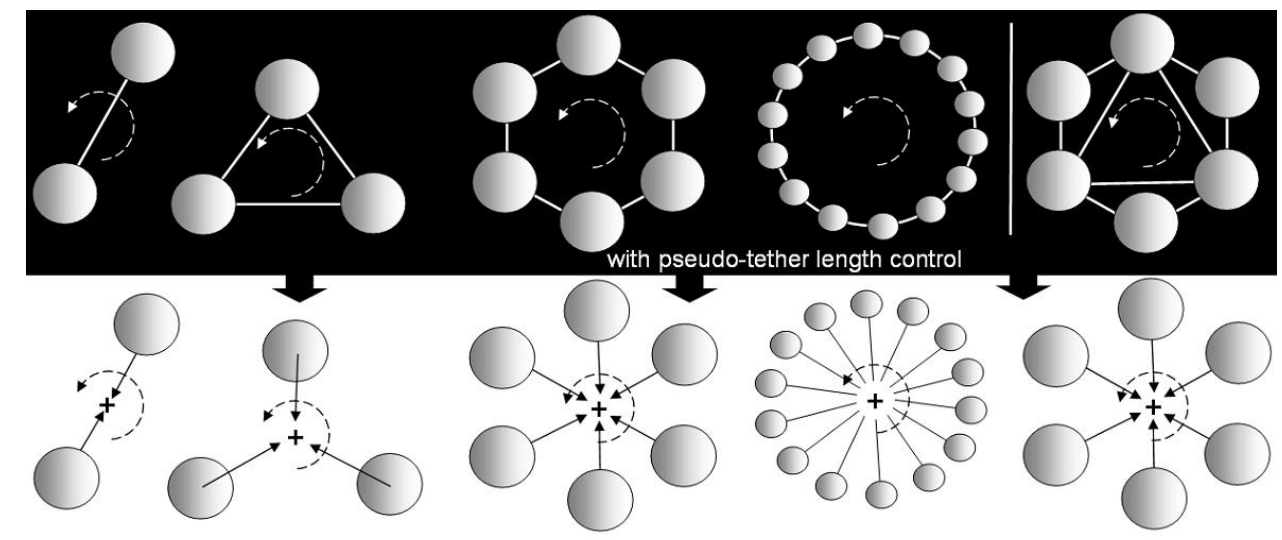

Figure 4. Reduction of circular or star tethered arrays into multiple single-tethered systems

\section{B. Three Inline Array}

Similarly, the dynamics of the three-spacecraft inline configuration in Figure 5 can be decoupled into two independent dynamics of the single-tethered system with the tether length $\ell$ and rotational dynamics of the center spacecraft. The equations of motions of the in-line array dynamics are developed using Lagrange's equation, 


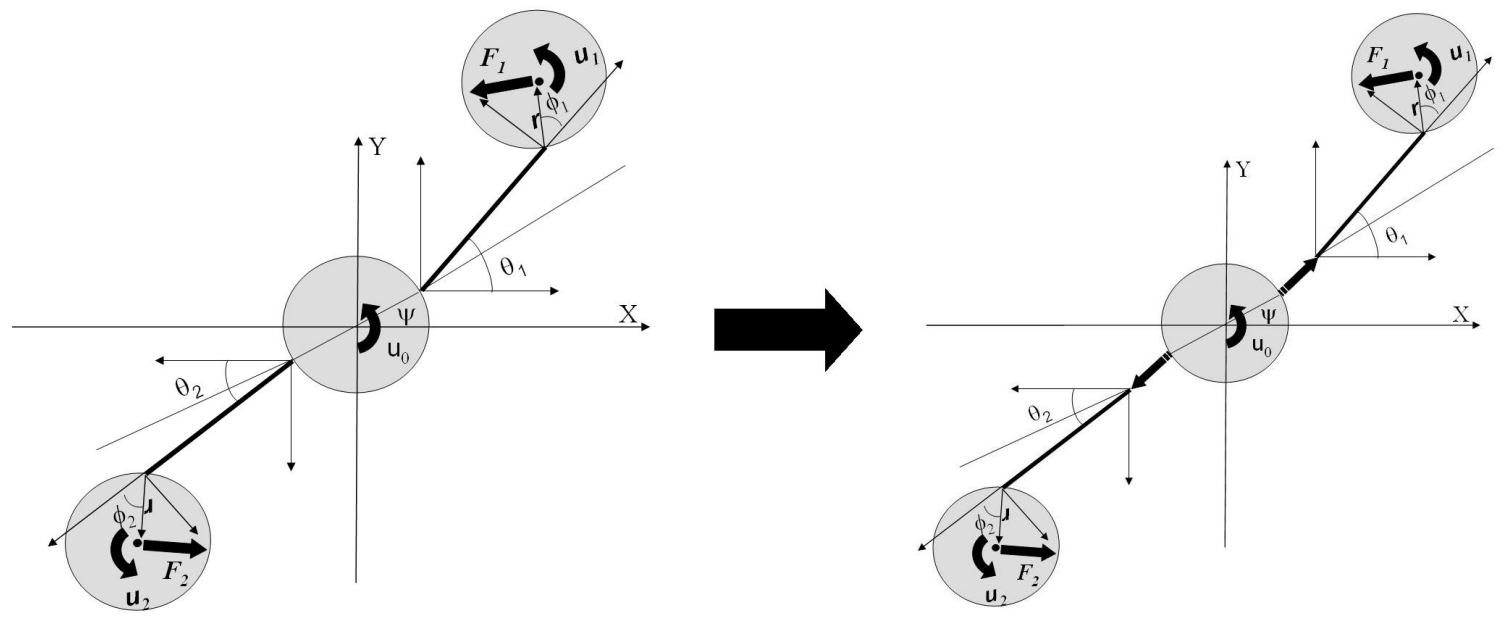

Figure 5. Three-spacecraft array decoupled into three individual dynamics

$\left[\begin{array}{ccccc}M_{11} & M_{12} & M_{13} & M_{14} & M_{15} \\ M_{12} & \mathbf{M}_{\mathbf{1}}\left(\phi_{1}\right) & 0 & 0 \\ M_{13} & 0 & 0 & 0 \\ M_{14} & 0 & 0 & \mathbf{M}_{\mathbf{1}}\left(\phi_{2}\right) \\ M_{15} & 0 & 0 & \end{array}\right]\left(\begin{array}{c}\ddot{\psi} \\ \ddot{\theta_{1}} \\ \ddot{\phi_{1}} \\ \ddot{\theta_{2}} \\ \ddot{\phi_{2}}\end{array}\right)+\left[\begin{array}{ccccc}0 & C_{12} & C_{13} & C_{14} & C_{15} \\ C_{21} & \mathbf{C}_{\mathbf{1}}\left(\phi_{1}, \dot{\theta_{1}}, \dot{\phi}_{1}\right) & 0 & 0 \\ C_{31} & 0 & 0 \\ C_{41} & 0 & 0 & \mathbf{C}_{\mathbf{1}}\left(\phi_{2}, \dot{\theta_{2}}, \dot{\phi}_{2}\right)\end{array}\right]\left(\begin{array}{c}\dot{\psi} \\ C_{51} \\ \dot{\theta_{1}} \\ \dot{\phi_{1}} \\ \dot{\theta_{2}} \\ \dot{\phi_{2}}\end{array}\right)=\left(\begin{array}{c}u_{0} \\ \tau_{\theta, 1} \\ \tau_{\phi, 1} \\ \tau_{\theta, 2} \\ \tau_{\phi, 2}\end{array}\right)$

where $2 \times 2$ matrices, $\mathbf{M}_{\mathbf{1}}\left(\phi_{1}\right)$ and $\mathbf{C}_{\mathbf{1}}(\phi, \theta, \dot{\phi})$ are from the single-tethered dynamics in figure 2 and Eq (1). In addition,

$M_{11}=I_{r}=I_{G}+2 m r^{2}$,

$M_{12}=m r \ell \cos \left(\theta_{1}-\psi\right)+m r^{2} \cos \left(\theta_{1}+\phi_{1}-\psi\right)$,

$M_{13}=m r^{2} \cos \left(\theta_{1}+\phi_{1}-\psi\right)$,

$M_{14}=m r \ell \cos \left(\theta_{2}-\psi\right)+m r^{2} \cos \left(\theta_{2}+\phi_{2}-\psi\right)$,

$M_{15}=m r^{2} \cos \left(\theta_{2}+\phi_{2}-\psi\right)$,

$C_{12}=-m r \ell \sin \left(\theta_{1}-\psi\right) \dot{\theta_{1}}-m r^{2} \sin \left(\theta_{1}+\phi_{1}-\psi\right)\left(\dot{\theta_{1}}+\dot{\phi}_{1}\right)$,

$C_{13}=-m r^{2} \sin \left(\theta_{1}+\phi_{1}-\psi\right)\left(\dot{\theta}_{1}+\dot{\phi}_{1}\right)$,

$C_{14}=-m r \ell \sin \left(\theta_{2}-\psi\right) \dot{\theta}_{2}-m r^{2} \sin \left(\theta_{2}+\phi_{2}-\psi\right)\left(\dot{\theta}_{2}+\dot{\phi}_{2}\right)$,

$C_{15}=-m r^{2} \sin \left(\theta_{2}+\phi_{2}-\psi\right)\left(\dot{\theta}_{2}+\dot{\phi}_{2}\right)$,

$C_{21}=m r \ell \sin \left(\theta_{1}-\psi\right) \dot{\psi}+m r^{2} \sin \left(\theta_{1}+\phi_{1}-\psi\right) \dot{\psi}$,

$C_{31}=m r^{2} \sin \left(\theta_{1}+\phi_{1}-\psi\right) \dot{\psi}$,

$C_{41}=m r \ell \sin \left(\theta_{2}-\psi\right) \dot{\psi}+m r^{2} \sin \left(\theta_{2}+\phi_{2}-\psi\right) \dot{\psi}$,

$C_{51}=m r^{2} \sin \left(\theta_{2}+\phi_{2}-\psi\right) \dot{\psi}$.

Note that these equations are simpler than those with a different coordinate definition of $\theta$, e.g., $\theta$ defined with respect to the rotating frame of the center spacecraft $(\theta \rightarrow \theta+\psi)$. Moreover, this definition of coordinates enables decoupling of the dynamics of the outlying spacecraft from the center spacecraft, as seen in the figure.

The dynamics of the spacecraft at the tips of the array are

$$
\begin{aligned}
& \left(\begin{array}{c}
M_{12} \\
M_{13}
\end{array}\right) \ddot{\psi}+\left(\begin{array}{c}
C_{21} \\
C_{31}
\end{array}\right) \dot{\psi}+\mathbf{M}_{\mathbf{1}}\left(\phi_{1}\right)\left(\begin{array}{c}
\ddot{\theta}_{1} \\
\ddot{\phi}_{1}
\end{array}\right)+\mathbf{C}_{\mathbf{1}}\left(\phi_{1}, \dot{\theta_{1}}, \dot{\phi}_{1}\right)\left(\begin{array}{c}
\dot{\theta_{1}} \\
\dot{\phi}_{1}
\end{array}\right)=\left(\begin{array}{c}
\tau_{\theta, 1} \\
\tau_{\phi, 1}
\end{array}\right) \\
& \left(\begin{array}{c}
M_{14} \\
M_{15}
\end{array}\right) \ddot{\psi}+\left(\begin{array}{c}
C_{41} \\
C_{51}
\end{array}\right) \dot{\psi}+\mathbf{M}_{\mathbf{1}}\left(\phi_{2}\right)\left(\begin{array}{c}
\ddot{\theta}_{2} \\
\ddot{\phi_{2}}
\end{array}\right)+\mathbf{C}_{\mathbf{1}}\left(\phi_{2}, \dot{\theta_{2}}, \dot{\phi}_{2}\right)\left(\begin{array}{c}
\dot{\theta_{2}} \\
\dot{\phi_{2}}
\end{array}\right)=\left(\begin{array}{c}
\tau_{\theta, 2} \\
\tau_{\phi, 2}
\end{array}\right)
\end{aligned}
$$

This is a hierarchical combination, as introduced in Theorem 2 since the dynamics reduce to those of the 
single-tethered system when the dynamics of $\psi$ vanish. It is easy to implement an exponentially stabilizing controller for $\psi$, in particular with measurement of tether tensions. ${ }^{24}$ So, as long as $\ddot{\psi} \rightarrow 0, \psi \approx \theta_{i}$, and $\phi_{i} \rightarrow 0$ (or $\ddot{\psi}, \dot{\psi} \rightarrow 0$ ), the same controller stabilizing the single-tethered system in the previous sections will also stabilize the combined system. Eq. (28) then verifies the following,

$$
\mathbf{M}_{\mathbf{1}}\left(\phi_{1}\right)\left(\begin{array}{c}
\ddot{\theta_{1}} \\
\ddot{\phi_{1}}
\end{array}\right)+\mathbf{C}_{\mathbf{1}}\left(\phi_{1}, \dot{\theta_{1}}, \dot{\phi}_{1}\right)\left(\begin{array}{c}
\dot{\theta_{1}} \\
\dot{\phi_{1}}
\end{array}\right)-\tau_{\mathbf{1}}=\mathbf{M}_{\mathbf{1}}\left(\phi_{2}\right)\left(\begin{array}{c}
\ddot{\theta_{2}} \\
\ddot{\phi_{2}}
\end{array}\right)+\mathbf{C}_{\mathbf{1}}\left(\phi_{2}, \dot{\theta_{2}}, \dot{\phi}_{2}\right)\left(\begin{array}{c}
\dot{\theta_{2}} \\
\dot{\phi_{2}}
\end{array}\right)-\tau_{\mathbf{2}}
$$

According to the synchronization theorem (Theorem 3), $\theta_{1}$ and $\phi_{1}$ exponentially converge to $\theta_{2}$ and $\phi_{2}$, respectively as long as the decentralized controllers, $\tau_{\mathbf{1}}$ and $\tau_{\mathbf{2}}$ make each single-tethered system contracting. In other words, contraction analysis shows that implementing a control law based upon the single-tethered dynamics ensures the stability of the rotational rate and the relative motions in an inline three-spacecraft array.

\section{Simulation and Experimental Results}

\section{A. SPHERES testbed}

The SPHERES testbed ${ }^{23,24}$ was developed as part of the ongoing research initiatives of the MIT Space Systems Laboratory (MIT-SSL) that utilizes the space environment provided by the space shuttle and International Space Station (ISS) to validate dynamics and control algorithms of distributed spacecraft control, estimation, and autonomy algorithms. The operational environments also include the 2-dimensional flat floor facilities at MIT and the NASA Marshall Space Flight Center.

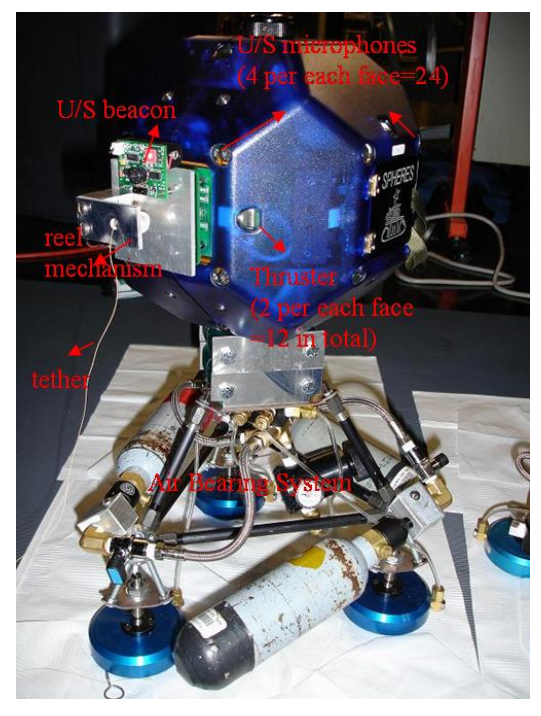

(a) SPHERES with previous air-carriage and tether reel

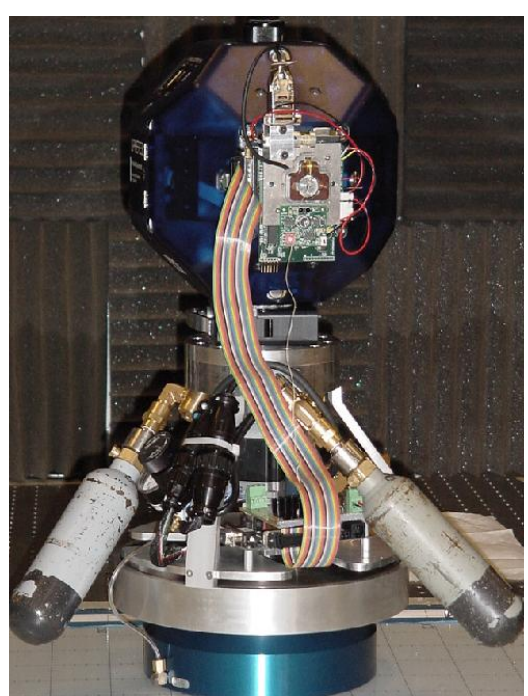

(b) SPHERES with new air-carriage and a reaction wheel

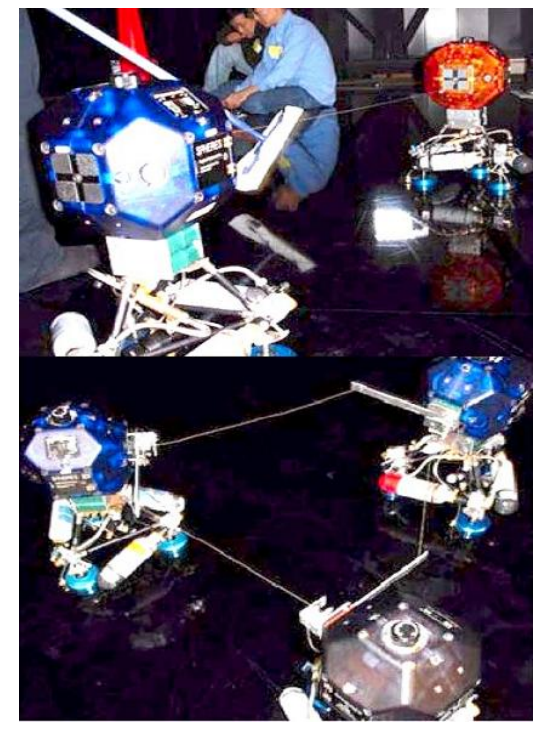

(c) Tethered SPHERES on the NASA MSFC flat floor

Figure 6. SPHERES tether experimental setup

The individual self-contained satellites (figure 6) have the ability to maneuver in up to six degrees of freedom, to communicate with each other and with the laptop control station, and to identify their position with respect to each other and to the experiment reference frame. The diameter of a single SPHERES is $0.25 \mathrm{~m}$, and the mass is $4.18 \mathrm{~kg}$. The satellites are propelled by a cold-gas thruster system which uses carbon dioxide as propellant. The CO2 propellant is stored in liquid form at 860 psig; a regulator reduces the pressure to 35 psig. Twelve thrusters are positioned to provide controllability in all six degrees of freedom, enabling both torque and translation control. Each thruster assembly consists of a solenoid-actuated microvalve with machined nozzles. The SPHERES metrology system using the ultrasound ranging system and gyroscope, provides metrology information to the satellites in real-time. Since no global metrology system like GPS is actually available in deep space missions, the tethered SPHERES system utilizes a relative 
metrology system using four ultrasound receivers (24 in total) on the line-of-sight face and the on-board beacon of the adjacent SPHERES (see figure 6). The relative metrology system is a pseudo-GPS ranging system that uses ultrasonic time-of-flight measurements from the target on-board beacon to the ultrasonic microphones distributed on the surface of each satellite. These time-of-flight measurements are converted to ranges and are then used to derive relative attitude $(\phi)$ with respect to the reference frame using a series of Extended Kalman Filters (EKF). An additional Kalman filter incorporating the gyroscope measurement estimates all the states needed $(\dot{\theta}, \dot{\phi}, \phi)$ for each satellite. Each estimation algorithm is decentralized in the sense it uses the single-tethered dynamics in Eq. (1). Texas Instruments C6701 Digital Signal Processor provides the computational power. A FLASH memory size of $224 \mathrm{~KB}$ allows software re-configuration of the full operating system, ensuring that multiple investigators are supported while the system is in the ISS. The tether deployment and retraction mechanism ${ }^{24}$ with tether tension sensors has been added to support the tethered formation mission.

\section{B. Simulation Results for Three Inline Spacecraft}

A controller introduced in Eq. (15) $\left(\tau=\mathbf{M}_{\mathbf{1}}(\mathbf{q}) \ddot{\mathbf{q}}_{\mathbf{r}}+\mathbf{C}_{\mathbf{1}}(\mathbf{q}, \dot{\mathbf{q}}) \dot{\mathbf{q}}_{\mathbf{r}}-\mathbf{K}\left(\dot{\mathbf{q}}-\dot{\mathbf{q}}_{\mathbf{r}}\right)\right)$ is used to spin up the two spacecraft at the tip of the inline three-spacecraft array in Figure 5 while a simple control law, $u_{0}=K(\omega-\dot{\psi})$ spins the center spacecraft. The desired spin rates, $\dot{\theta}_{1 d}, \dot{\theta}_{2 d}, \dot{\psi}_{d}$ are $\omega=0.3 \mathrm{rad} / \mathrm{s}$ with the minimal compound pendulum mode. The initial rotational rates $\left(\dot{\theta}_{10}, \dot{\theta}_{20}, \dot{\psi}_{0}\right)$, are $0.2,0.22,0.2 \mathrm{rad} / \mathrm{s}$ with the zero compound pendulum modes, $\phi_{1}, \dot{\phi_{1}}, \phi_{2}, \dot{\phi_{2}}=0$. The radius of SPHERES, $r$ is $0.125 \mathrm{~m}$, the tether length, $\ell$ is $1 \mathrm{~m}$, the mass of SPHERES with the air-bearing carriage $(\mathrm{m})$ is $8.6 \mathrm{~kg}$, and the moment of inertia, $I$ is $0.074 \mathrm{kgm}^{2}$. Figure 7 shows that a controller from the single spacecraft dynamics successfully performs to spin up the array to the desired rate.

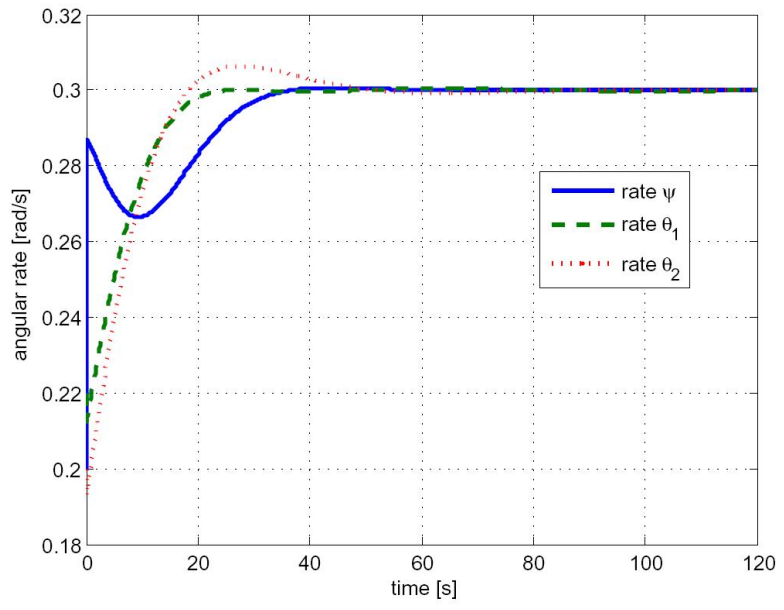

(a) Array angular rate

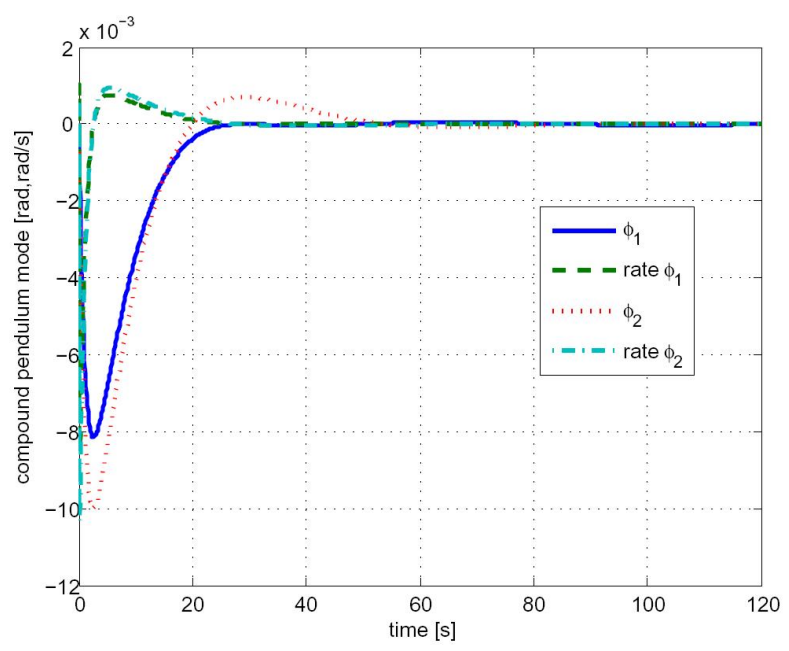

(b) Compound pendulum mode

Figure 7. Simulation results for the inline three configuration.

\section{Experimental Results for Two-Tethered System}

A nonlinear control approach based on Input-State Feedback Linearization ${ }^{16}$ is employed when the system is fully actuated (i.e both thruster force $F$ and torque $u$ are available as control input)

This computed torque controller is given as the following:

$$
\tau_{\mathbf{1}}=\mathbf{M}_{\mathbf{1}}(\mathbf{q})\left(\ddot{\mathbf{q}}_{\mathbf{d}}-2 \boldsymbol{\Lambda} \dot{\tilde{\mathbf{q}}}-\boldsymbol{\Lambda}^{2} \dot{\tilde{\mathbf{q}}}\right)+\mathbf{C}_{\mathbf{1}}(\mathbf{q}, \dot{\mathbf{q}}) \dot{\mathbf{q}}
$$

which is equal to the controller in Eq. (15) with $\mathbf{K}=\mathbf{M}_{\mathbf{1}} \mathbf{\Lambda}$ and $\dot{\mathbf{q}} \rightarrow \dot{\mathbf{q}}_{\mathbf{r}}$. Likewise, the controller for the second spacecraft can be similarly defined.

If the target angular rate is $\omega$ and the objective of the control is to spin up the interferometric array to $\omega$ while minimizing the compound pendulum mode, the desired trajectory is defined as follows: $\dot{\theta}_{d}=$ 
$\left(\omega-\dot{\theta}_{0}\right)\left(1-e^{-\tau t}\right)+\dot{\theta}_{0}, \ddot{\theta}_{d}=\left(\omega-\dot{\theta}_{0}\right) \tau e^{-\tau t}, \ddot{\phi}_{d}=0, \dot{\phi}_{d}=0, \phi_{d}=0$. This will lead to an exponentially stable closed-loop dynamics, $\ddot{\tilde{\mathbf{q}}}+2 \boldsymbol{\Lambda} \dot{\tilde{\mathbf{q}}}+\boldsymbol{\Lambda}^{2} \dot{\tilde{\mathbf{q}}}=0$

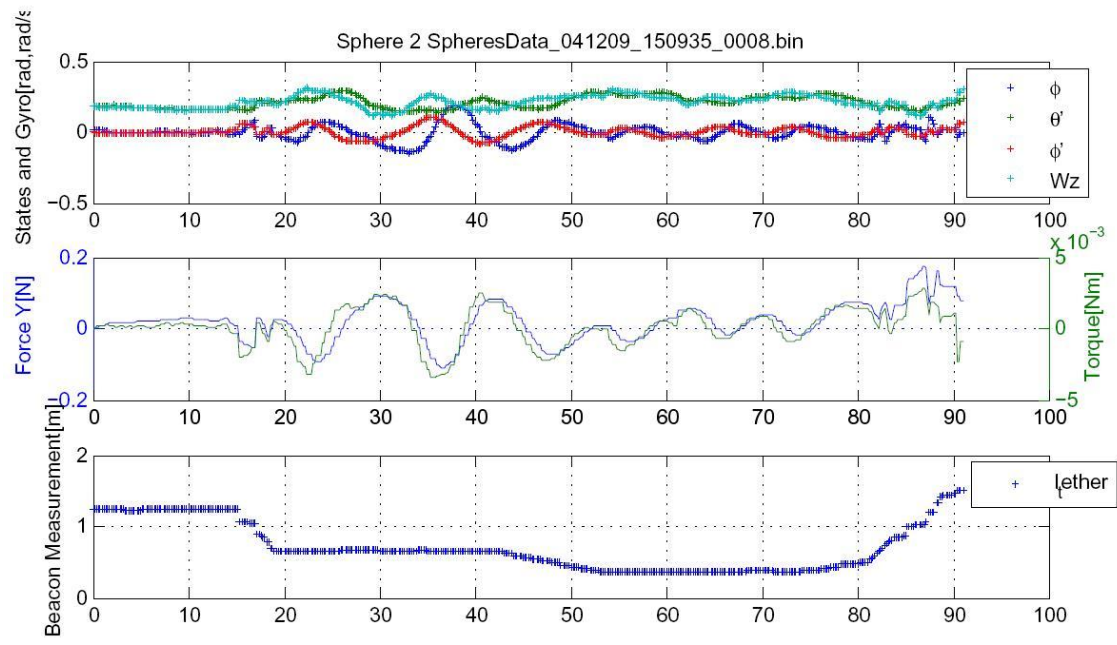

Figure 8. nonlinear controller with varying tether length in two tethered satellites, Sat2

Figure 8 shows the experimental data with this nonlinear controller collected from one of the SPHERES of a two-body formation (see Figure 6) The third row of the figures represents the tether length measured directly using the ultrasound ranging metrology system. It is observed that the compound pendulum mode $(\phi, \dot{\phi})$ gets excited when the tether reels in as predicted in II. Note that the effect of varying the tether length, or the speed, $\dot{\ell}$ was not considered in the nonlinear model. So a better performance is achievable by taking into account the motor speed $\dot{\ell}$. More experiments with a new air-carriage system will be conducted in July 2006.

\section{Conclusion}

We introduce a decentralized control technique by reducing the original dynamics to simpler stable closed-loop dynamics exploiting oscillation synchronization. Contraction theory proves that a decentralized control law can be designed based on a single-tethered system in lieu of the coupled dynamics of multiple spacecraft, reducing the computation and communication burdens. Furthermore, the stability of the combined system is global and exponential. The technique is extended to the three-spacecraft inline configuration and the triangular configuration, both of which are being studied for NASA's SPECS mission. Results of numerical simulations and experiments using the SPHERES testbed show the effectiveness of the proposed control strategy. The video clips of the experiments in this paper can be downloaded at http://ssl.mit.edu/spheres/videos.html.

\section{Acknowledgments}

The author is grateful to Prof. Jean-Jacques Slotine and Prof. David W. Miller for stimulating discussions.

\section{References}

${ }^{1}$ Chung, S.-J., Miller, D. W., deWeck, O. L., "ARGOS Testbed: Study of Multidisciplinary Challenges of Future Spaceborne Interferometric Arrays," SPIE Optical Engineering, Vol. 43, No. 9, pages 2156-2167, September 2004.

${ }^{2}$ Beichman, C. A., Woolf, N. J., and Lindensmith, C.A., "The Terrestrial Planet Finder (TPF): A NASA Origins Program to Search for Habitable Planets," JPL Publication 99-3, 1999.

${ }^{3}$ Leisawitz, D., et al. "Scientific Motivation and Technology Requirements for the SPIRIT and SPECS Farinfrared/Submillimeter Space Interferometers," Proceedings SPIE 4013, 2000, p. 36.

${ }^{4}$ Quinn, D. A., and Folta, D. C., "A Tethered Formation Flying Concept for the SPECS Mission," Proceedings of the 23rd 
Rocky Mountain Guidance and Control Conference, Vol. 104, Univelt, San Diego, CA, 2000, pp. 183-196.

${ }^{5}$ Strogatz, S., Sync: The Emerging Science of Spontaneous Order, New York: Hyperion, 2003.

${ }^{6}$ Kandel, E. R., et al., Principles of Neural Science, Fourth Edition, McGraw Hill.

${ }^{7}$ Bernstein, N., "The Coordination and Regulation of Movements," Perseus, 1967.

${ }^{8}$ Bizzi, E., et al., "Modular organization of motor behavior in the frog's spinal cord," Trends in Neurosciences Review 18:442-445

${ }^{9}$ Collins, J. J., and Stewart, I. N., "Coupled Nonlinear Oscillators and the Symmetries of Animal Gaits," Nonlinear Science, 3:349-392, 1993.

${ }^{10}$ Jadbabaie, A., Lin, J., and Morse, A. S. "Coordination of Groups of Mobile Autonomous Agents Using Nearest Neighbor Rules," IEEE Transactions on Automatic Control, May 2003.

${ }^{11}$ Shan, J., and Liu, H.-T., "Close-Formation Flight Control with Motion Synchronization," Journal of Guidance, Control, and Dynamics, Vol. 28, No. 6, 2005.

${ }^{12}$ Fowler, J. M., and D'Andrea, R., "A Formation Flight Experiment," IEEE Control Systems Magazine, October 2003.

${ }^{13}$ Krishnaprasad, P. S., and Tsakiris, D., "Oscillations, SE(2)-Snakes and Motion Control: A Study of the Roller Racer, " Dynamics and Stability of Systems, 2001.

${ }^{14}$ Belanger, G. M., et al., "Decentralized Control of Satellite Clusters Under Limited Communication," AIAA 2004-5023, AIAA GNC Conference, Providence, RI, 2004.

${ }^{15}$ Smith, R. S., and Hadaegh, F. Y., "Control of Deep-Space Formation-Flying Spacecraft; Relative Sensing and Switched Information," AIAA J. GCD, Vol. 28, No. 1 Jan-Feb 2005.

${ }^{16}$ Slotine, J.-J. E., and Li, W., Applied Nonlinear Control, Prentice Hall,. New Jersey, 1991.

${ }^{17}$ Lohmiller, W., and Slotine, J.-J E., "On Contraction Analysis for Nonlinear Systems," Automatica, 34(6), 1998.

${ }^{18}$ Slotine, J.J.E., "Modular Stability Tools for Distributed Computation and Control," Int. J. Adaptive Control and Signal Processing, 17(6), 2003.

${ }^{19}$ Wang, W., and Slotine, J.-J. E., "On Partial Contraction Analysis for Coupled Nonlinear Oscillators," Biological Cybernetics, 92(1), 2004.

${ }^{20}$ Lohmiller, W., and Slotine, J.-J. E., "Control System Design for Mechanical Systems Using Contraction Theory," IEEE Transactions on Automatic Control, Vol. 45, No. 5, May 2000.

${ }^{21}$ Slotine, J.-J. E., Wang, W., and El Rifai, K., "Synchronization in Networks of Nonlinearly Coupled Continuous and Hybrid Oscillators," Sixteenth International Symposium on Mathematical Theory of Networks and Systems (MTNS 2004), July 2004.

${ }^{22}$ Olfati-Saber, R. "Nonlinear Control of Underactuated Mechanical Systems with Application to Robotics and Aerospace Vehicles," Ph.D thesis, Department of EECS, MIT, February 2001.

${ }^{23}$ Kong, M. C., et al., "SPHERES as a Formation Flight Algorithm Development and Validation Testbed: Current Progress and Beyond," 2nd International Symposium on Formation Flying Missions and Technologies, September 14-16, 2004, Washington, DC.

${ }^{24}$ Chung, S.-J., Kong, E., and Miller, D. W., "Dynamics and Control of Tethered Formation Flight Spacecraft Using the SPHERES Testbed," Proceedings of AIAA Guidance, Navigation and Control Conference, San Francisco, August 2005

${ }^{25}$ Nakaya, K, et al., "Formation Deployment Control for Spinning Tethered Formation Flying - Simulations and Ground Experiments," AIAA GNC Conference, August 2004, AIAA 2004-4896.

${ }^{26}$ Beletsky and Levin, Dynamics of Space Tether Systems, American Astronautical Society Publication Vol. 83.

${ }^{27}$ Kojima, H., et al., "Nonlinear Control of Librational Motion of Tethered Satellites in Elliptic Orbits," J of Guidance, Control and Dynamics, Vol.27, No. 2, March-April 2004.

${ }^{28}$ Kokubun, K., et al., "Deployment,Retrieval Control of Tethered Subsatellite Under Effect of Tether Elasticity," J. of Guidance, Control and Dynamics, Vol.19,No.1, January-February 1996.

${ }^{29}$ Fujii, H. A., "Wave-Absorbing Control of Transverse Vibration of Tethered System," Journal of Astronautical Sciences, Vol 51, No.3, pp 249-259, 2004.

${ }^{30}$ Kim, M., and Hall, C. D., "Control of a Rotating Variable-Length Tethered System," J. of Guidance, Control and Dynamics, Vol.27, No.5, Sep-Oct 2004.

${ }^{31}$ Bombardelli, C., Lorenzini, E. C., Quadrelli, M. B., "Retargeting Dynamics of a Linear Tethered Interferometer," AIAA J. of Guidance, Control and Dynamics, Vol. 27, No.6, Nov-Dec 2004

${ }^{32}$ Kumar, K. D. and Yasaka, Y., "Rotating Formation Flying of Three Satellites Using Tethers," J. Spacecraft and Rockets, Vol. 41, No. 6, Nov-Dec 2004

${ }^{33}$ Bergamaschi, S., and Bonon, F., "Coupling of Tether Lateral Vibration and Subsatellite Attitude Motion," J. Guidance, Control, and Dynamics, Vol. 15, No, 5.

${ }^{34}$ Quadrelli, M. B., "Modeling and Dynamics Analysis of Tethered Formations for Space Interferometry," Paper AAS $01-231$.

${ }^{35}$ Misra, A. K., "Equilibrium Configurations of Tethered Three-Body Systems And Their Stability," Paper AAS 01-191.

${ }^{36}$ M. Bennett, M. F. Schatz, H. Rockwood, and K. Wiesenfeld, "Huygens's clocks," in Proc. (A) of the Royal Society, 2001.

${ }^{37}$ Bullo, F., and Lewis, A. D., Geometric Control of Mechanical Systems- Modeling Analysis, and Design for Simple Mechanical Control Systems, Texts in Applied Mathematics, Springer-Verlag, 2004.

${ }^{38}$ Chang, D. E., and Marsden, J. E., "Reduction of Controlled Lagrangian and Hamiltonian Systems with Symmetry," SIAM J. CONTROL OPTIM.,Vol. 43, No. 1, pp. 277 - 300.

${ }^{39}$ Crassidis, J.L., and Junkins, J.L., Optimal Estimation of Dynamic Systems, CRC Press, Boca Raton, FL, 2004.

${ }^{40}$ Miller, D.W. and Grocott, S.C., "Robust control of the Multiple Mirror Telescope adaptive secondary mirror," Optical Engineering 38(08), p. 1276-1287. 